\title{
LA FIGURA DE LA SUSPENSIÓN DE LA EJECUCIÓN DE LA PENA EN LAS LEGISLACIONES EUROPEAS. ESPECIAL REFERENCIA A LA "PENA" DE PROBATION
}

Chloé Lafarge Turpín ${ }^{1}$

\begin{tabular}{|c|c|}
\hline INFORMACIÓN DEL ARTÍCULO & RESUMEN \\
\hline $\begin{array}{l}\text { Historia del artículo: } \\
\text { Recibido el } 1 \text { de octubre de } 2018 \\
\text { Aceptado el } 19 \text { de marzo de } 2019\end{array}$ & $\begin{array}{l}\text { El presente trabajo se propone estudiar la configuración de la figura de } \\
\text { la suspensión en el ámbito europeo. Con este fin, se hace hincapié en } \\
\text { primer lugar en las dos instituciones pioneras que condujeron a la }\end{array}$ \\
\hline $\begin{array}{l}\text { Palabras claves: } \\
\text { Suspensión de la ejecución de la pena } \\
\text { Reinserción social } \\
\text { Derecho europeo } \\
\text { Pena privativa de libertad }\end{array}$ & $\begin{array}{l}\text { definición de sus contornos legales actuales: la probation y el sursis. A } \\
\text { continuación, se hace especial referencia a la figura de la probation } \\
\text { autónoma, una pena en "vía de desarrollo" en el conjunto del espacio } \\
\text { europeo y que, probablemente, podría pasar a constituir en la próximas } \\
\text { décadas una potente herramienta de individualización de la pena de } \\
\text { cara a imponer al delincuente la respuesta judicial más susceptible de } \\
\text { favorecer su reeducación y reinserción social. }\end{array}$ \\
\hline
\end{tabular}

THE FIGURE OF THE SUSPENSION OF THE EXECUTION OF THE PENALTY ON THE EUROPEAN LEGISLATIONS. SPECIAL REFERENCE TO PROBATION PENALTY

\section{ABSTRACT}

\section{Keywords}

Suspension of the penalty execution Imprisonment

Social reintegration

European law
The aim of this article is to study the configuration of the penalty suspension in the european area. In order to complete this target, we insist, first of all, on the two pioneers institution that lead to its actual definition : the probation and the «sursis ». Then, we make a reference to the «autonomous» probation, a «developing penalty » in various european countriesthat could be, in the next decades, a powerful tool of individualization of the penalty in order to impose on the offender the judicial answer more susceptible to stimulate his social rehabilitation.

${ }^{1}$ Doctora en Derecho. Universidad de Zaragoza, España. lafarge.turpin@gmail.com 


\section{LA SUSPENSIÓN DE LA EJECUCIÓN DE LA PENA EN EUROPA: FIGURAS PIONERAS}

En primer lugar, cabe insistir en que el estudio del régimen de suspensión de la ejecución de la pena en la legislación de los países europeos supone el de las dos figuras pioneras que condujeron a la definición de los contornos legales de la suspensión de la pena, el de la probation y el belga-francés del sursis. Se trata de los dos mecanismos que, a pesar de haberse desarrollado de forma bastante distinta, introdujeron en la legislación de numerosos países la posibilidad de suspender la pena de prisión de corta duración.

\subsection{Probation y sursis}

El primer uso de la probation se fijó en Boston, en 1841, cuando un artesano llamado John Augustus se levantó ante un Tribunal para ofrecerse para prestar fianza y supervisar a un hombre acusado de embriaguez común (Maqueda, 1985). Sin embargo, Maqueda (1985) afirma que en el año 1820, ciertos magistrados de Warwichshire, en Inglaterra, ya habían implementado una práctica propiamente probatoria, al someter a varios delincuentes que tenían bajo su control a un régimen de prueba y vigilancia ${ }^{2}$. Este intento empezó a reiterarse a partir del año 1841, cuando algunos jueces de Inglaterra y Estados Unidos pasaron a autorizar la libertad de condenados, bajo ciertas condición y vigilancia, con el fin de evitar los efectos negativos de la prisión (Maqueda, 1985; Araújo, 2009). De tal modo "que estas prácticas se constituyeron en factores históricos embrionarios, que permitieron desarrollar la idea de vigilancia y, consecuentemente, el instituto de la probation" (Araújo, 2009, p. 233). Casi 30 años más tarde se dio el siguiente paso al aprobar en

\footnotetext{
${ }^{2}$ Maqueda (1985) afirma que "en todo caso, no puede ignorarse su presencia, ya iniciado el siglo XIX, como exigencia indispensable para el "tratamiento en libertad" de pequeños infractores y jóvenes delincuentes. Un sector minoritario de la doctrina señala en 1820 el ensayo de una práctica propiamente
}

1869 el estado de Massachusetts, un estatuto con la provisión de un agente estatal para investigar casos de niños sometidos a prueba ante los tribunales. Sin embargo, la probation solo adquirirá su "moderna estructuración y desarrollo" (Maqueda, 1985, p. 50) a finales del siglo XIX con "The Massachusetts Statute on Probation" en 1878 y el "Summary Jurisdiction Act" inglés en 1897.

Entrado ya el siglo XX, esta institución ya empezó a ser recogida y desarrollada en los sistemas norteamericanos e ingleses, dando lugar a la aparición del régimen de prueba conocido hoy en día como modelo anglosajón, mientras que paralelamente, iba emergiendo en Europea el que iba a ser el otro gran sistema de referencia en materia de probation, el sursis.

En primer lugar, es importante señalar que, a pesar de constituir estas dos instituciones las "creaciones jurídicas pioneras" (Araújo, 2009, p. 229) que hicieron posible la suspensión de la pena de prisión de corta duración, también existen otros sistemas que se acercan a estas dos opciones. Así, Cobo y Vives (1991) afirman que existen otras figuras similares "en cuanto a sus resultados, pero diferentes en orden a su naturaleza" (p. 851), como la amonestación con reserva de pena (Verwarnung mit Strafvorbehalt). También mencionan instituciones que se aproximan a esos sistemas, como la dispensa de pena, el perdón judicial o la compensación, que según Cobo y Vives (1991) representan "auténticas causas de exclusión de la pena" (p. 851). Por lo que respecta a la amonestación con reserva de pena alemana, se trata de una forma de suspensión de fallo (Araújo, 2009) mediante la cual se "reconoce la culpabilidad del acusado, pero se suspende la imposición de la

probatoria a instancia de ciertos magistrados de Warwichshire (Inglaterra) que procedieron a aplicar un estricto régimen de vigilancia sobre los delincuentes que tenían bajo su competencia, para lo cual disponían de un registro de personas de confianza a las que asignar esa misión" (p. 50). 
pena, recibiendo el autor una advertencia" (Prat, 2004 , p. 40). En el caso de delinquir el sujeto dentro de los tres años siguientes a esta advertencia, se le impondrá una multa, pudiendo también fijarse algunas condiciones. Se trata no obstante de una figura prevista para delitos castigados con pena de multa y no con prisión (Serrano, 1999). En cuanto a la figura española de suspensión, cabe recordar que el Proyecto de Código penal de 1980 introducía en sus artículos 91 y siguientes, la suspensión de fallo. Se trataba entonces de dejar al órgano jurisdiccional la posibilidad de dictar la sentencia suspendiendo el fallo (Gracia y Alastuey, 2006), por un periodo de dos a cinco años. Para Serrano (1999), este mecanismo se acercaba sin duda a la condena condicional, puesto que, durante el plazo establecido, la suspensión estaba condicionada a que el sujeto, bajo el control del Juez de Vigilancia Penitenciaria y observando determinadas reglas de conducta, no volviera a delinquir. De esta forma, una vez cumplido el plazo señalado respetando las condiciones impuestas, no constaría en nombre del culpado ningún antecedente penal. Sin embargo, esta suspensión del fallo, de inspiración anglosajona (Gracia y Alastuey, 2006) no fue retenida ni en el proyecto de $1980 \mathrm{ni}$ el Anteproyecto de Código Penal de 1983, al optar el legislador por la remisión condicional. Afirmó así Serrano (1999) que "los mayores problemas se refirieron a la mala educación de nuestro sistema procesal a esa especie de doble instancia que parece presuponer la suspensión del fallo: de un lado, el pronunciamiento de la culpabilidad y, de otro, el pronunciamiento de la condena" (p. 291).

\subsubsection{El sistema anglosajón}

\section{$\underline{\text { Rasgos específicos }}$}

Tal y como mencionamos en el apartado anterior, una de las principales características de esta institución consiste en que a pesar de ser reconocida la responsabilidad penal del sujeto por el juez u órgano sentenciador, no se pronuncia la sentencia, siendo sometido el sujeto a un periodo de prueba vigilado. En consecuencia, si el delincuente cumple con las condiciones establecidas por el Juez, la pena jamás será pronunciada (Jescheck y Weigend, 2003). Gracias y Alastuey (2006) afirman al respecto que:

"el Juez o Tribunal, una vez que haya declarado la culpabilidad del encausado, interrumpe el proceso, no pronunciando la sentencia ni imponiendo en consecuencia la pena en la misma, a fin de someter al reo a un periodo de prueba; durante este periodo el sujeto está obligado a la observancia de las reglas de conducta que le haya fijado el Juez o Tribunal y queda sometido a la vigilancia y tutela de los delegados de prueba; en el caso de que el reo no haya delinquido durante ese periodo de prueba, el Juez o Tribunal no dictará nunca la sentencia y, por ello, no le queda al reo ningún antecedente penal" (p. 295) ${ }^{3}$.

Otra peculiaridad de este sistema consiste en que se puede reconocer y declarar la culpabilidad del delincuente en momentos distintos. Una vez que el sujeto ha sido declarado culpable, es decir constatada la culpabilidad, Núñez (2005) explica que:

"el juez suspende el pronunciamiento de la condena e impone una serie de "obligaciones" o "cargas" de carácter educacional o resocializador al imputado que éste deberá observar durante un determinado periodo de tiempo, aunque bajo el control, dirección y asistencia, bien del propio Tribunal, bien del "agente de prueba" (probation officer). Transcurrido el

\footnotetext{
${ }^{3}$ En el mismo sentido, J. Barquín (citado en Araújo, 2009).
} 
periodo de tiempo predeterminado, el reo debe comparecer de nuevo ante el tribunal, que a la vista del cumplimiento o no de las condiciones que se establecieron, acuerda dejar sin efecto la condena o pronunciamiento del fallo" (p. 706).

Así, esta diferenciación procesal permite al juez valorar si el simple reconocimiento de la culpabilidad del sujeto es suficiente para alcanzar efectos preventivos-especiales, sin necesidad de pasar por la aplicación de una pena de prisión (Araújo, 2009; Marín (2000). Efectivamente, Araújo (2009) recuerda que:

"se considera que la mera declaración de culpabilidad puede ser más eficaz que la propia amenaza de una pena. Por este motivo, la pena solo se impondrá al delincuente en el caso de que no aproveche las posibilidades de resocialización que se le ofrecen" (p. 235).

También hace falta mencionar aquí la peculiaridad de algunos territorios de Estados Unidos en los que la probation puede sustituir el reconocimiento de la culpabilidad con el fin de evitar las consecuencias nocivas de la condena (Núñez, 2005). En ese sentido, Núñez (2005) señala que:

"el juez puede suspender el pronunciamiento de la condena o ejecución de la pena, aunque este último sistema no se aplica en la práctica en cuanto es considerado contrario a la finalidad esencialmente resocializadora de la probation, ya que impone al delincuente el estigma de la condena y le carga con unos

\footnotetext{
${ }^{4}$ Este autor hace referencia, además, a una "diversidad de matices probatorios complementarios de imposición facultativa de reglas de conducta en el Proyecto de 1980 y de prognosis en el Propuesta de 1983, que parece acercarse, aunque tímidamente, a las disposiciones legislativas de los países continentales tales como el sursis del Derecho francés, con
}

antecedentes penales que pueden dificultar su rehabilitación" (p. 740)

Vemos entonces que estas características no se asemejan al procedimiento común europeo en el que la declaración de culpabilidad y la imposición de la pena suelen aparecer unidos.

\subsubsection{Determinación y contenido}

En primer lugar, la probation se caracterizará por la imposición, durante un determinado periodo de tiempo, de condiciones y reglas de conducta destinadas a favorecer la reinserción social del delincuente. Se podrá tratar de condiciones muy variadas, adaptadas al sistema jurídico de cada Estado (Maqueda, 1985).

En segundo lugar, una de las dimensiones más relevantes de esta medida es sin duda la de control y vigilancia, una labor que forma parte integrante del sistema de probation "desde su aparición en el contexto político penal y que hacen aún hoy de ella una medida funcional respecto de las estructuras características del sistema socio-económico y político actual" (Maqueda, 1985, p.229). Se trata de una tarea que, según Marín (2000), es "imprescindible para concretar la reinserción del delincuente en la sociedad, ya que es por medio de estas acciones que se lleva a cabo el tratamiento necesario a la recuperación del criminal" (p. 744). En esta óptica, se desarrollarán actividades como "la de vigilancia, de asistencia y de orientación al inculpado con el fin de que pueda superar los problemas que se presenten durante su readaptación social" (Marín, 2000, p. 744), informándose al órgano jurisdiccional de forma regular "acerca de la evolución del reo y de los resultados de la prueba" (Araújo, 2009, p. 238).

imposición de règles de conduite del Derecho suizo, o la Strafaussetzung zur Bewährung del Derecho alemán, en cuanto que dichas disposiciones establecen "instrucciones", "obligaciones" o "reglas de conducta", con la supervisión, ayuda o asistencia del agente de prueba". 
Esta labor de control y vigilancia constituye la diferencia más importante y más substancial Judel y Piñol citado en Suárez, 2002) entre el sistema anglosajón y, como detallaremos a continuación, el sistema de sursis. Desempeña un papel imprescindible y deberá ser llevada a cabo por un profesional especializado: el agente de prueba o "probation officer", que establecerá un contacto profesional, personal y neutral (Maqueda, 1987) con el penado con el objetivo de guiarle mediante una relación de confianza hacia una reinserción social definitiva (Maqueda, 1987). Este seguimiento se aleja mucho de simples diligencias policiales o para-policiales destinadas a la comprobación de buena conducta durante el periodo de prueba (Araújo, 2009). El control y la vigilancia se convierten así en "significativas formas de tratamiento" (Araújo, 2009, p. 238), "una interferencia directa y firme en la personalidad, en el modo de vida y en el sentido moral del culpado" (Maqueda, 12987, p. 231). La vigilancia adquiere aquí "la dimensión precisa que se espera de ella, la de un "tratamiento fundado sobre las ciencias sociales y psicológicas y practicado conscientemente como una actividad profesional" que se ejerce dentro de un cuadro de control gradual" (Araújo, 2009, p. 239).

Por fin, también será necesaria la emisión de un pronóstico sobre la viabilidad o no de realizar un tratamiento, una característica que pone sin duda de manifiesto la dimensión individualizadora de la probation (Araújo, 2009). Así, toda decisión de probation deberá ir precedida por un análisis riguroso, de carácter científico, social, psicológico y médico (Maqueda, 1987) que posibilite la clasificación del sujeto en una categoría criminológica establecida, lo que se conoce como "diagnosis", para sobre esta base "emitir el juicio de pronóstico correspondiente $\mathrm{y}$, respecto del cual, la opción de probation se hará depender de la decisión "científica" acerca de la necesidad o no de tratamiento" (Maqueda, 1987, p. 232).

En cuanto a la valoración de este sistema, amplio es el sector doctrinal que se muestra favorable a la implantación del modelo anglosajón de probation. Sin embargo, algunos autores matizan esta voluntad al afirmar que este sistema presenta varios problemas específicos. De allí que, según Barquín (2007):

"aspectos tales como la indeterminación legal de las condiciones a imponer, así como el peso excesivo que puede tener en la valoración del periodo de prueba el delegado de probation constituyen una importante traba a la hora de implantar dicho sistema en ordenamientos jurídicos donde el principio de legalidad constituye una barrera formalmente infranqueable" ( $p$. 237).

Por el contrario, no consideramos que el papel acordado al agente de probation sea incompatible con el principio de legalidad. Este funcionario informa al juez con frecuencia acerca del desarrollo del periodo de prueba y de la evolución personal del penado. Se trata entonces de una labor de seguimiento bastante elaborada, que cuenta con una estrecha colaboración entre el órgano jurisdiccional y el personal social y educativo responsables de la ejecución de las medidas. El hecho de que el juez conozca en profundidad la situación del delincuente, generalmente fiable en razón de la buena formación de los agentes, y pueda adaptar la duración y forma de ejecución del periodo de prueba a esta realidad no nos parece en absoluto un obstáculo a la legalidad de la medida. El juez sigue determinando el contenido de la pena y el trabajo desempeñado por los agentes de probation solo contribuye a hacer más efectiva la individualización de la pena. 
En suma, el sistema anglosajón de probation merece una valoración positiva, al procesar firmemente la individualización de la pena y favorecer el cumplimiento de los fines preventivas especiales de la pena (Gracia y Alastuey, 2006; Maqueda, 1987; Judel y Piñol citado en Suárez, 2002; Araújo, 2009). La figura del probation officer constituye sin duda, a nuestro modo de ver, el futuro de toda medida de suspensión o alternativa a la pena de prisión, al constituir un apoyo imprescindible y un eficaz intermedio entre las instituciones sociales y jurisdiccionales. Finalmente, también es de alabar el hecho de que esta medida no limite el ejercicio de los derechos políticos y laborales y evite que consten antecedentes en nombre del penado (Maqueda, 1987).

\subsection{El sursis belga-francés}

Antes de interesarnos por las diferencias sustanciales existentes entre la medida de sursis y el sistema anglosajón de probation, cabe aquí hacer referencia a sus aspectos y fines comunes. Efectivamente, ambos "se proponen instrumentalizar las condiciones legales necesarias con el fin de evitar los efectos negativos de la pena de prisión" (Araújo, 2009, p. 243) a la vez que constituyen herramientas de "lucha frente a las penas cortas de privación de libertad" (Maqueda, 1985, p. 56). Según Maqueda (1985), el sursis o suspensión condicional:

"no presentaba una fisionomía idéntica a la probation cuando apareció en Europa. Probablemente su único punto de enlace era la finalidad común que, en un principio, inspiraba a ambas: sustraer el ambiente criminógeno de la prisión a una determinada categoría de delincuentes" ( $p$. 55).

Aunque pretendan alcanzar fines idénticos, el sursis se diferencia del sistema anglosajón en varios aspectos. En primer lugar, en el marco de una medida de sursis, el órgano jurisdiccional dictará el veredicto de culpabilidad del delincuente para imponerle su consecuencia jurídica, es decir, la pena privativa de libertad. Así, se suspenderá luego la ejecución de una pena ya impuesta (Gracia y Alastuey, 2006) y no el fallo, como sería el caso en el sistema anteriormente estudiado. Según palabras de Jescheck y Weigend (2003), "no solo es comprobada la culpabilidad, sino que también se falla la pena a imponer, aunque su ejecución es suspendida condicionalmente" (p. 87). De esta forma, si el penado no vuelve a delinquir en el plazo de suspensión establecido la pena originalmente impuesta será definitivamente remitida. En segundo lugar, en el sursis simple, no es frecuente la imposición de reglas de conducta o condiciones distintas de no volver a delinquir (Jescheck y Weigend, 2003). La vigilancia y el seguimiento del penado no son entonces características propias del sursis.

La principal diferencia entre los dos sistemas estudiados reside entonces en el hecho de que en la probation se interrumpen el pronunciamiento de la sentencia y la imposición de la pena mientras que en el sursis se suspende la ejecución de la pena (Araújo, 2009). Maqueda (1985) pone así de manifiesto que la declaración de culpabilidad y el pronunciamiento de la sentencia son uno de los elementos que "definen el sursis continental y permiten distinguirlo, en una primera aproximación, de instituciones afines, tales como la probation anglo-americana (suspensión de la sentencia condenatoria y no solo de la pena)". Este sistema tendrá entonces como consecuencia la permanencia de antecedentes penales tras el cumplimiento del plazo de suspensión, lo que será "decisivo a efectos de una posterior reincidencia; durante el periodo de prueba, el reo no goza de ninguna asistencia ni se le dispensa ningún tratamiento" (Gracia y Alastuey, 2006, p. 300). 
La tendencia actual en el continente europeo (Araújo, 2009) consiste en optar por un modelo híbrido, que consiste generalmente en suspender la pena y someter el condenado a prueba (Araújo, 2009). Este modelo mixto supone la:

"suspensión condicional de la pena con sometimiento a prueba, en el que se pretende compensar con uno las carencias del otro. Se trata de la solución imperante en el Derecho continental europeo que, si bien coincide prácticamente con el modelo del sursis simple, se diferencia de ésta en que, al igual que hace la probation, otorga a los jueces la posibilidad de someter el condenado, aparte de a la obligación de no delinquir, a otros tipos de deberes o de controles que el sujeto suspendido también deberá cumplir durante un determinado periodo de suspensión fijado en sede judicial si quiere ver finalmente remitida su pena" (J. Barquín citado por Araújo, 2009, p. 244).

No obstante, el análisis de la legislación de los países europeos también nos llevó a constatar que varios de ellos configuran la probation como una pena y no como una alternativa a la pena privativa de libertad derivada de la suspensión de la pena o de su fallo. El análisis comparado de la configuración de esta figura nos permitió poner de manifiesto sus principales características.

\section{LA MODERNA "PENA" DE PROBATION: CONFIGURACIÓN}

La figura de la probation considerada como pena ya es de aplicación en varios países europeos. Antes de proceder al examen de la legislación de cada uno de ellos, intentaremos destacar los principales rasgos de un modelo general.

En primer lugar, cabe insistir en que la pena de probation se configura como una medida "autónoma" que no depende ni se apoya en una pena de prisión. No existe esa "espada de Damocles" (Tournier, 2013) que, como en el caso de la suspensión, puede ser ejecutada si el penado comete un nuevo ilícito penal o vulnera una de las reglas de conducta o medidas impuestas por el juez. De esta forma, solamente se articula alrededor de un periodo de prueba realizado en la comunidad (Tournier, 2013) y constituye una pena más entre las ya existentes en el ordenamiento jurídico. Tal y como afirmó la jurista francesa Dindoh (2011), en el momento de la introducción de esta pena en la legislación francesa:

"sería muy interesante que una persona pudiera ser condenada a una medida de probation disociada de la pena de prisión y que la no ejecución de la medida constituya otro delito, de la misma forma que se imponen condenas por no realizar un trabajo en beneficio de la comunidad o por fugarse en el caso de cumplir una pena de prisión. Cuando un penado se fuga de la cárcel, parece lógico que vuelva a ser condenado. Cuando no cumplen las medidas impuestas por la probation, se trata de otro tipo de evasión, ¿por qué no volver a ser condenado?" (p. 14).

La no ejecución o quebrantamiento de las medidas inherentes a la pena conduce así a una nueva comparecencia ante el juez que decide acerca de las nuevas obligaciones, prohibiciones o pena a imponer.

Por otra parte, la configuración de la pena de probation no suele ser sinónima de la desaparición de las figuras alternativas a la prisión como la suspensión o la sustitución de la pena. Tal y como veremos a continuación, sistemas penales como el del Reino Unido, incluyen la pena de probation así como varias modalidades de suspensión, incluso la suspensión del fallo. Somos conscientes de que un abanico tan amplio de respuestas penales necesita una rigurosa regulación de sus requisitos de 
aplicación y que parece imprescindible limitar la arbitrariedad del juez haciendo corresponder cada figura a perfiles criminológicos bien delimitados. La elección entre dos instituciones cercanas, como podrían ser por ejemplo la pena de probation y la suspensión del fallo, resultaría compleja en ausencia de criterios de concesión claros y bien fundamentos, dirigidos obviamente a obtener los mejores efectos preventivo-generales. A pesar de esta exigencia, concordamos aquí con un amplio sector doctrinal según el cual la suspensión tanto de la ejecución de la condena como del fallo podría coexistir con esta nueva forma de probation, reservándose las primeras para infracciones de mayor gravedad (Dindoh, 2011; Tournier, 2013; Dintilhac, 2013; Bouvier, 2013). La probation autónoma constituye así una posibilidad de mayor individualización de la pena a la vez que permite ampliar y afinar las distintas respuestas penales ya existentes en el ámbito de los delitos menos graves.

Tomando como referencia la situación penitenciaria de Francia, Tournier (2007) afirmaba que:

"las penas privativas de libertad representan el $55 \%$ de las sanciones pronunciadas. Sin embargo, el $63 \%$ de estas penas se suspenden. De esta forma, la cárcel constituye la pena de referencia sin serlo (suspensión) y siéndolo a la vez (riesgo de revocación)" (p. 3) ${ }^{5}$.

En este contexto, este autor hacía hincapié en el hecho de que la pena de probation podría sustituir la pena de prisión en numerosos supuestos y proponía entonces su introducción en la legislación francesa afirmando que su aplicación podría representar por lo menos $1 / 3$ de las penas y superar las penas de prisión (20\%). Consideraba así que ciertas infracciones castigadas en aquel momento con una pena privativa de libertad podían ser objeto de la nueva probation y que esta pena permitiría acercar la legislación penal francesa a lo preconizado por el Consejo de Europa, haciendo pasar el porcentaje de "sanciones aplicadas en la comunidad" del $18 \%$ al 40\% (Tournier, 2007). Opinamos aquí que el uso de la pena de probation puede efectivamente contribuir a la disminución del número de condenas a pena de prisión. Si viene acompañada, por ejemplo, de la realización de trabajos en beneficio de la comunidad, esta pena puede alcanzar una capacidad preventiva general alta y un poder preventivo-especial más eficaz que la pena de prisión. Se podría así disminuir la cantidad de delitos susceptibles de ser castigados con la cárcel y esperar la obtención de mejores indicios de no reincidencia. No se trata entonces de reducir el número de ingresos en prisión a través del aumento de las concesiones de suspensión o sustitución de la pena de prisión sino mediante la disminución de la posibilidad de imponerla. En suma, la regulación de la pena de probation permite limitar la lista de delitos castigados con la pena de prisión y no supone la introducción de un mecanismo alternativo más, destinado a contrarrestar sus efectos nocivos. A nuestro modo de ver, su uso conjunto con instituciones como la sustitución o la suspensión permitiría el logro de mejores resultados en el ámbito de la prevención especial.

En cuanto al plazo de cumplimiento de la pena de probation, suele fijarse en el momento de su imposición y no es susceptible de volver a sufrir ninguna modificación antes de su finalización (Tournier, 2013). En función de la naturaleza de la infracción cometida $y$ de la situación $y$ circunstancias personales del sujeto, la probation

${ }^{5}$ Ver capítulo $\mathrm{V}$, relativo al carácter preventivo general y especial del TBC. 
podrá suponer la imposición de distintas reglas de conducta, se trate de obligaciones, como por ejemplo la de someterse a un tratamiento médico o a una actividad de interés general, de prohibiciones, como la de acercarse a un determinado lugar o a una determinada persona, la de practicar ciertas actividades o de medidas de vigilancia, tanto físicas como electrónicas (Tournier, 2013). Estas condiciones, determinadas por el órgano jurisdiccional, podrán, a diferencia de la duración del periodo de prueba, verse modificadas durante la ejecución de la medida (Dindoh, 2011; Torunier, 2013; Dintilhac, 2013; Bouvier, 2013). Se plantea entonces aquí la delicada cuestión del correcto reparto de las competencias en cuanto a la determinación de las modalidades y objetivos de seguimiento del penado. En este contexto, Bishop (2012) aseguró que:

"un criterio esencial de la severidad de las sanciones es el grado de intrusismo, de intervención, permitido por el Estado en la vida del delincuente. Existen diferencias en el nivel de intervención en la vida del delincuente entre las distintas sanciones aplicadas en la comunidad. Comparen, por ejemplo, el trabajo en beneficio de la comunidad y la libertad vigilada bajo control telemático. En consecuencia, a mi modo de ver, el Tribunal debe decidir a la vez acerca de la duración de la sanción y de su naturaleza y para poder tomar tales decisiones, es necesario poder disponer de un informe pre-sentencia. Este mecanismo es el recogido por la regla 31 de las Reglas europeas relativas a las sanciones $y$ medidas aplicadas en la comunidad (R92/16). El informe pre-sentencia puede basarse en una evaluación de los riesgos, es lo que se lleva a cabo en Suecia, desde hace varios años".

Al contrario, otras voces se elevan para explicar que con el fin de asegurar "una real individualización de la pena, la jurisdicción solo fijará la duración del periodo de prueba. El agente de probation, tras evaluar la situación personal del sujeto, será el competente para determinar los objetivos y las modalidades del seguimiento. A partir de las propuestas realizadas por el agente, la autoridad judicial se pronunciará acerca de eventuales restricciones de libertad" 6 . A nuestro modo de ver, tal y como desarrollamos en el anterior epígrafe, la fuerte implicación del agente de probation en la determinación del contenido de la pena no supone ninguna vulneración del principio de legalidad, hace posible un seguimiento eficaz del penado así como la imposición por parte del juez de una medidas de ejecución del periodo de prueba más adaptadas a sus circunstancias personales. Estamos de acuerdo con Bishop (2012), al considerar que el Tribunal es el encargado de definir tanto la duración de la sanción como su contenido. Sin embargo, esta definición deber ser el resultado de una colaboración estrecha y continua con los servicios sociales y no de un único informe pre-sentencia, a menudo elaborado en un corto periodo de tiempo e insuficiente para plasmar la situación personal, laboral y social del penado.

Por lo que respecta al quebrantamiento de las condiciones de probation, las distintas legislaciones estudiadas coinciden en que el sujeto tendrá que volver a comparecer ante el Juez que impuso la medida. Este último podrá modificar el contenido de la sanción inicialmente impuesta o imponer otra de distinta naturaleza, que podrá

\footnotetext{
${ }^{6}$ Comunicado del XXVII Congrès du Syndicat national de l'ensemble des personnels de l'administration pénitentiaire, Paris, diciembre de 2012.
} 
consistir en una pena de prisión. No obstante, a diferencia de lo que ocurriría en el marco de la probation o de la suspensión ordinaria, esta última se impondrá por presentar un supuesto interés de cara a la reinserción social del individuo y no por defecto, al revocarse la suspensión de una pena ya pronunciada 0 al reanudarse un proceso interrumpido. Se tratará de examinar las causas de la vulneración de las condiciones impuestas y de actuar conforme con las necesidades del delincuente. El hecho de que no haya sido capaz de someterse a las características de la medida de probation no significa que la pena de prisión será adaptada y más eficaz para alcanzar su reinserción social.

Por otra parte, parece que esta configuración como "pena" se acerca al concepto de probation contenido en la normativa europea, en particular la Recomendación del Consejo de Europa de 20 de enero de 2010. Esta última describe la probation como "la ejecución en medio abierto de sanciones y medidas definidas por ley y pronunciadas contra el autor de una infracción". Consiste así en "una serie de actividades e intervenciones que suponen el seguimiento, consejo y apoyo con el fin de reinsertar socialmente el autor de la infracción y de contribuir a la seguridad colectiva". En esta óptica, la recomendación fija las características del proceso de supervisión de los infractores que se materializará mediante distintas fases. Así, con el fin de analizar de forma sistemática y exhaustiva, antes y durante la ejecución de la medida, la situación particular del penado, "incluyendo los riesgos, los factores positivos y necesidades, las intervenciones necesarias para abordar las mismas, así como una estimación de la receptividad del infractor a estas intervenciones" 7 , la "valoración" tendrá como principal objetivo la

${ }^{7}$ Recomendación CM/Rec(2010)1 del Comité de Ministros a los Estados miembros sobre las reglas del Consejo de Europa relativas a la probation, Ministerio del Interior, art. 66, pág. 13. ${ }^{8}$ Idem, art. 72 , p. 14 determinación del tipo de intervención más adaptado para cada sujeto. Esta primera fase será seguida por la realización de un "plan de ejecución de penas y medidas" que "guiará la labor de los Servicios de Probation, permitiendo al personal y a los propios infractores evaluar el progreso hacia los objetivos establecidos" 8 . La fase de planificación debe entonces conducir, de acuerdo con el infractor, a la fijación de modalidades de resolución de los problemas identificados durante el proceso de valoración. En cuanto a la fase de intervención está destinada a poner en práctica lo previamente decidido y acordado, al tener por "objeto la rehabilitación y la renuncia al delito, debiendo por lo tanto ser constructivas y proporcionales a la pena o medida impuesta" ${ }^{9}$. Sin entrar en el detalle de los distintos tipos de intervenciones, la Recomendación precisa que los "Servicios de Probation podrán recurrir a utilizar una variedad de métodos basados en un enfoque interdisciplinario y en conocimientos sólidos derivados de la investigación pertinente"10. Por fin, la fase de evaluación implica que "los progresos de los infractores se evaluarán a intervalos regulares, repercutiendo en el plan de ejecución durante el resto de la supervisión" 11 y que "la evaluación revelará igualmente el grado en que el plan de ejecución ha sido definido, aplicado y ha producido los resultados deseados" 12 .

A nuestro modo de ver, este concepto de probation, configurada como pena, más centrado en el seguimiento $y$ apoyo al penado $y$ no dependiente de otro procedimiento penal ya abierto puede potenciar su eficacia a nivel educativo, criminológico o respecto a su reinserción social (Tournier, 2013; Dintilhac, 2013; Bouvier, 2013). En primer lugar, permite ampliar el número de respuestas penales a la comisión del

\footnotetext{
9ldem, art. 76, p. 14.

10 dem, art. 77, p. 14.

11 dem, art. 81 , p. 15.

12 Idem, art. 82, p. 15.
} 
delito. La probation podría constituir una pena destinada a castigar delitos de escasa gravedad cuyo autor necesite especial apoyo y acompañamiento de cara a alcanzar la reinserción social. Así, se podría posibilitar su imposición en el caso de perfiles criminológicos necesitados de un seguimiento más personalizado y en el que la capacidad preventiva-especial de las penas alternativas tradicionales podría resultar insuficiente. En suma, la pena de probation permite afinar la individualización de la pena y acercarse a lo dispuesto por las recomendaciones europeas que establecen que el juez debe disponer de un catálogo amplio de penas alternativas en el que pueda elegir la sanción que, teniendo en cuenta la proporcionalidad con el delito cometido, contrarreste mejor los factores que pueden llevar al sujeto a cometer nuevos delitos ${ }^{13}$.

De la misma manera, también podría permitir la disminución de la imposición de la pena de prisión puesto que el mayor recurso a la suspensión de la ejecución de la pena no supone una disminución del uso de la pena de prisión sino que solo impide el inicio de su ejecución. Se podría así conceder una alta capacidad preventivo-especial ${ }^{14}$ a la pena a la vez que se lograría la atenuación del uso sistemático de la pena de prisión y la estigmatización inherente a ella. Esta última solo será susceptible de ser aplicada en caso de quebrantamiento de las condiciones de mantenimiento de la pena cuando el juez lo estime necesario en relación con las circunstancias

\footnotetext{
${ }^{13}$ Recommendation (92) 16, European rules on community sanctions and measures, 19 octubre 1992, regla 5 y Recommendation (2000) 22, Improving the implementation on the European rules on community sanctions and measures, 29 noviembre 2000, apéndice 2 , arts. 1 y 2 .

14 Ver Capítulo V, la realización de trabajos en beneficio de la comunidad, medida frecuentemente impuesta en el marco de la pena de probation es susceptible de producir altos efectos resocializadores mediante varios mecanismos, en particular: el compromiso de la comunidad, la valoración positiva del trabajo realizado por el condenado, la percepción de la justicia
}

particulares del reo y los motivos que le condujeron a volver a delinquir.

Por otra parte, la introducción de esta figura debería ser sinónima, al igual que en los Estados que la recogen en su legislación, de la existencia de un Cuerpo profesional especializado, dedicado exclusivamente a asegurar la supervisión y el acompañamiento de los penados en la realización de sus actividades. La creación de semejante órgano especializado permitiría, a nuestro modo de ver, acabar con el concepto de supervisión entendido como tarea de control ${ }^{15}$ y potenciar el poder rehabilitador de las medidas alternativas.

\section{LA "PENA" DE PROBATION EN LAS LEGISLACIONES EUROPEAS}

Recogemos en la siguiente tabla los datos obtenidos durante el estudio de la legislación de los principales países europeos en relación con la posible existencia de una pena de probation.

\subsection{Alemania}

El Código penal alemán no incluye la probation entre las penas aplicables al autor de un ilícito penal. Solamente recoge unas medidas "de control y de asistencia" (die Führungsaufsicht) cuya ejecución se llevará a cabo bajo la supervisión de un agente (Bewährungshelfer) del servicio de probation (Aufsichtsstelle). Estas obligaciones podrán ser impuestas por el juez como:

\section{pena accesoria;}

procesal, la adquisición de técnicas y conocimientos y la intervención sobre las necesidades criminógenas.

${ }^{15}$ Según la Recomendación (2010) 1, sobre Reglas de Probation, la supervisión de la persona en la comunidad por parte de un agente de probation no debe consistir solo en una tarea de control sino que debe proporcionar a los infractores consejo, ayuda y motivación para dejar de delinquir y debe combinarse, cuando sea necesario, con intervenciones dirigidas a mejorar la formación de la persona, sus habilidades personales y sus oportunidades de empleo. 
en caso de suspensión de una pena de prisión, 0 ;

como medida de seguridad cuando el penado se encuentre en libertad condicional ${ }^{16}$.

\subsection{Francia}

Desde octubre de 2014, el sistema francés prevé la figura de la contrainte pénale ("sumisión penal"), una pena "independiente, sin vinculo ni referencia a la pena de prisión" (Dintilhac, 2013, p. 132). El art. 131-8-2 del Código penal recoge entonces una medida autónoma, destinada a ser aplicada de forma independiente, como una pena más del arsenal jurídico (Bouvier, 2013) y que "no está directamente "adosada" a una pena de prisión" (Dintilhac, 2013, p. 132; Bouvier, 2013, p. 133). Supone la imposición de medidas de control y de determinadas obligaciones cuya ejecución se llevará a cabo bajo la supervisión del "Juez de aplicación de las penas" Juge de l' application des peines) (Tournier, 2013) durante un periodo de entre seis meses y cinco años. En cuanto a la naturaleza de las obligaciones y prohibiciones susceptibles de ser impuestas, el art. 131-8-2 recoge: las medidas de control aplicables en los supuestos de suspensión de la pena (art. 132-44 $\mathrm{CP}$ ); la obligación de realizar un trabajo en beneficio de la comunidad (hasta 210 horas de trabajo en beneficio de una entidad pública o de una asociación), según lo previsto en el art. 131-8 CP y el sometimiento a un tratamiento terapéutico.

Desde el 1 de enero de 2017, la contrainte pénale podrá imponerse cuando "la personalidad y la situación material, familiar y social del delincuente, así como el ílicito penal cometido justifiquen un seguimiento socio-educativo individualizado específico" 17 . Entendemos entonces que se posibilita la aplicación de esta

${ }^{16}$ Ver Strafgesetzbuch, StGB, Título IV, art. 56 y ss.

17 Según el art. 131-4-1 del Código penal francés, "lorsque la personnalité et la situation matérielle, familiale et sociale de l'auteur d'un délit puni d'une peine d'emprisonnement et les pena en el ámbito de cualquier delito cuando el juez lo estime oportuno con el fin de favorecer la reinserción social del sujeto. Se trata de un importante cambio en la legislación penal francesa puesto que dicha pena solo podía aplicarse en relación con los delitos castigados con una pena de prisión inferior a 5 años.

El Juez de aplicación de las penas determinará las obligaciones y prohibiciones más adaptadas a la situación del penado en función de los resultados de la evaluación de su personalidad por parte del servicio penitenciario de inserción y de probation. En caso de quebrantamiento de estas medidas, se podrá reforzar e intensificar las obligaciones inicialmente impuestas por el juez o pronunciar una pena de prisión cuya duración no podrá exceder de dos años.

Esta pena se diferencia en primer lugar de las medidas alternativas por tener un objetivo distinto y complementario de estas últimas. En efecto, según Herzog (2013), debe reservarse para determinados perfiles, necesitados de un real seguimiento y para los cuales la problemática a tratar en materia de prevención de la reincidencia y de reinserción justifica un acompañamiento pluridisciplinar. La probation autónoma se diferencia entonces de las penas alternativas que suelen suponer el control del condenado sin plantear la necesidad de un seguimiento individualizado (Herzog, 2013). También se aleja del concepto de "sursis" al favorecer la identificación de las personas necesidades de un seguimiento personal más intenso y en numerosos casos, pluridisciplinar. Así, afirma Dindoh (2011) que "el "sursis" debería requerirse cuando el control estricto del respeto de las obligaciones e interdicciones parece suficiente y la pena de

faits de l'espèce justifient un accompagnement socio-éducatif individualisé et soutenu, la juridiction peut prononcer la peine de contrainte pénale". 
probation cuando un seguimiento y acompañamiento individualizado parecen necesarios" (p. 90 y ss.).

\subsection{Italia}

El 2 de abril de 2014, la Camera dei deputati aprobó un proyecto de ley autorizando al Gobierno para regular las condiciones de ejecución de las penas alternativas a la cárcel y destinado a insertar en el Código penal varias disposiciones relativas al régimen de suspensión de la pena. La elaboración de esta reforma, que entró finalmente en vigor en abril de 2015 supuso la inserción, por una parte, de tres nuevos artículos, 168 bis a 168 quater en el Título IV "De la extinción del crimen y de la pena" del primer libro del Código penal titulado "De los crímenes en general" y por otra parte de ocho artículos, de 464 bis a 464 novies en el libro VI, dedicado a los procedimientos especiales del Código procesal penal. También dispone que el ministro de Justicia se verá obligado a comunicar al Parlamento cualquier necesidad de modificación de las estructuras encargadas de llevar a cabo estas medidas.

\subsubsection{Requisitos de aplicación de la suspensión del proceso}

Según la legislación vigente, el juez podrá ordenar "la suspensión del proceso con medida de probation" (sospensione del procedimiento con messa alla prova) cuando así lo solicite el acusado de forma oral o por escrito y presente un programa de tratamiento elaborado por el servicio de ejecución de las penas o en su ausencia la solicitud de elaboración de tal programa. El órgano judicial ordenará así la suspensión del proceso cuando considere que el programa se adapta a las circunstancias personales del acusado y que el desarrollo del tratamiento permite a la vez la protección de la víctima. Este programa, elaborado por el servicio de ejecución de las penas y cuyo contenido debe ser aprobado por la persona objeto de la suspensión del proceso prevé: las modalidades de participación del sujeto y de sus familiares, su situación social actual y sus distintas posibilidades de reinserción cuando se puedan determinar; las obligaciones y prohibiciones específicas a las que tendrá que someterse el penado (trabajo en beneficio de la comunidad, reparación del daño causado,...) y las medidas destinadas a promover, cuando sea posible su realización, la mediación con la víctima.

La solicitud de aplicación de tal medida solamente podrá intervenir en relación con delitos castigados con pena de multa o con pena de prisión inferior a cuatro años así como en los casos de agresión o amenaza a la autoridad pública, desacato a un magistrado, violación de sellos, riña con agravante y robo con receptación. Por otra parte, según los artículos 102, 105 y 108 del Código penal, la suspensión del proceso no podrá acordarse más de una vez ni respecto a "delincuentes habituales" o "delincuentes profesionales". En cuanto al desarrollo del periodo de probation, el servicio de probation tendrá la obligación de informar al juez, cada trimestre o al ritmo previsto por el órgano judicial, acerca de la actividad y del comportamiento de la persona objeto de la medida de suspensión, proponiendo si lo considera necesario modificaciones del programa, ya se trate de flexibilizar el contenido de las obligaciones impuestas o de reforzarlo.

Con el fin de facilitar su decisión respecto a la concesión de esta medida, el juez podrá obtener, mediante la intervención de la policía, de los servicios sociales o de cualquier otra entidad pública, todas las informaciones consideradas como necesarias en relación con las circunstancias personales y sociales del sujeto. Unos datos que serán inmediatamente comunicadas al Ministerio fiscal y al encargado de la defensa del acusado.

\subsubsection{El contenido de la medida de probation}


La medida de probation podrá implicar:

la realización de un trabajo en beneficio de la comunidad, adaptado a la profesión y a las aptitudes del delincuente. El trabajo en beneficio de la comunidad consistirá en una prestación no remunerada, de una duración mínima de 10 días y su realización se adaptará a las obligaciones familiares, profesionales o de formación del acusado, con un máximo de 8 horas al día;

el desempeño de tareas destinadas a la eliminación de las consecuencias perjudicables o peligrosas de la comisión del delito;

la reparación a la víctima de los prejuicios ocasionados por la comisión del delito;

la colaboración con entidades sociales en la realización de unas actividades de carácter social, la imposición de obligaciones respecto a determinados servicios sociales o estructuras de cuidados específicos u obligaciones relativas al lugar de residencia del penado, su libertad de circulación o la prohibición de acudir de ciertos lugares.

\subsubsection{Desarrollo y ejecución}

\section{Efectos}

La suspensión del proceso tiene como principales efectos la suspensión de la prescripción del delito y la sumisión del delincuente a obligaciones y prohibiciones determinadas por el juez. Si transcurre el periodo de probation sin vuelta a la delincuencia por parte del penado, la pena nunca llegará a pronunciarse, dejando al sujeto libre de antecedentes judiciales.

\section{Duración}

La duración de la suspensión del proceso no podrá superar los dos años para los delitos castigados con una pena de prisión, ya se trate de una pena inicial o de sustitución de una multa, y un año en el caso de delitos castigados con pena de multa.
Al finalizar este periodo, el juez declarará en una decisión específica la desaparición del delito, teniendo en cuenta los informas elaborados por el servicio de ejecución de las penas o la reanudación del proceso dejando de imponerse al condenado las obligaciones inicialmente impuestas.

\section{Modificación}

El plazo de ejecución de la medida determinado por el juez, solo podrá verse prorrogado una vez, si así lo solicita el penado alegando "motivos graves". También podrá modificar las condiciones de ejecución de la pena de probation, siempre que el penado dé su consentimiento y tras haber obtenido la opinión favorable del ministerio fiscal. Por fin, se podrá acordar el pago fraccionado del importe de la reparación de la víctima cuando esta se exige.

\section{Quebrantamiento de la medida}

La medida de suspensión del proceso será revocada en caso de vulneración grave o reiterada del programa de tratamiento o de las obligaciones impuestas o de negación por parte del penado de realizar el trabajo en beneficio de la comunidad. También se revocará al cometerse durante el periodo de probation, un delito que no tenga la consideración de imprudente (delito no colposo) o de un delito de idéntica naturaleza al que motivó la imposición de la probation. El ministerio fiscal restará de la nueva pena impuesta los días de probation efectivamente ejecutados, haciendo equivaler tres días de suspensión a un día de prisión o a una multa de 250 euros.

A modo de síntesis, cabe destacar en primer lugar que Italia dispone de un sistema de probation autónoma, entendida además como "suspensión del proceso" y no como suspensión del fallo. Se trata de la única legislación europea en hacer intervenir esta medida tan "temprano", puesto que entendemos que la determinación de la pena, momento procesal generalmente suspendido en la 
probation autónoma interviene posteriormente, o sea, al concluir el proceso judicial, un proceso que según el sistema aquí descrito no llega ni a desarrollarse por la decisión del Juez de acordar la medida de probation. En segundo lugar, también es relevante el hecho de que el acusado tenga la iniciativa de la solicitud de suspensión del proceso y elabore su propio plan de reinserción social en colaboración con el servicio de probation. A nuestro modo de ver, se trata sin duda de la forma más eficaz de obtener el pleno consentimiento del sujeto, tanto en relación con la imposición de la pena en sí misma como con su contenido. El proceso judicial y el proceso interno, personal y psicológico, que deriva de él se desarrollarán conforme a bases sólidas plenamente delimitadas y aceptadas por el acusado. Por fin, la ley italiana reconoce y valora la situación de la víctima, al hacer figurar en el programa elaborado por el delincuente y los servicios sociales, por una parte, medidas destinadas a promover la mediación con la víctima y por otra la obligación de averiguar si el lugar del domicilio del sujeto no constituye un obstáculo a la seguridad y protección de la víctima.

\subsection{Reino Unido}

\subsubsection{El Community order}

La legislación de Inglaterra y Gales recoge a la vez la pena de probation y la figura de la suspensión del fallo. La primera de ellas, introducida en el Criminal Justice Act en 2003, consiste en la imposición de un Community order, es decir de una pena a ejecutar en la comunidad. Esta medida solo se impondrá cuando el órgano jurisdiccional

\footnotetext{
18 Art. 1771 Criminal Justice Act 2003: "Where a person aged 10 or over is convicted of an offence, the court by or before which he is convicted may make an order imposing on him any one or more of the following requirements:

(a)an unpaid work requirement;

(b)an activity requirement;

(c)a programme requirement;

(d)a prohibited activity requirement;

(e)a curfew requirement;
}

tenga la convicción de que se adapta a la situación personal del acusado y que su contenido es proporcional a la gravedad del delito cometido. La medida deberá contener por lo menos una de las obligaciones e prohibiciones contenidas en el artículo 177 de la Ley penal de $2003^{18}$, es decir:

- la obligación de realizar un trabajo no remunerado;

- la obligación de desempeñar una actividad específica;

- la prohibición de desempeñar una actividad específica;

- la obligación de respetar un determinado horario;

- la prohibición de acudir a determinados lugares;

- la obligación de residir en un determinado sitio

- la prohibición del salir del territorio nacional-

- la obligación de someterse a un tratamiento psiquiátrico:

- la obligación de someterse a un tratamiento de desintoxicación de drogas;

- la obligación de someterse a un tratamiento de desintoxicación de alcohol;

- la obligación de someterse a un servicio de probation.

Para garantizar la aplicación de ciertas de estas obligaciones y prohibiciones, el Juez podrá prever el seguimiento telématico del acusado mediante pulsera electrónica ${ }^{19}$.

En junio de 2013, el London Probation Trust, el servicio de probation más importante de Inglaterra y Gales publicó una guía, el Benchguide to

\footnotetext{
(f)an exclusion requirement;

(g)a residence requirement;

(h)a mental health treatment requirement;

(i)a drug rehabilitation requirement;

(j)an alcohol treatment requirement;

(k)asupervisionrequirement".

19 Se impondrá por ejemplo en caso de restricción de horarios o de prohibición de acudir a determinados sitios.
} 
Community Sentences, destinada a ayudar a los magistrados en la determinación de las medidas a aplicar. Esa guía preconizaba para cada una de la obligaciones y prohibiciones aplicables, una determinada duración en función de la gravedad de la infracción cometida. Así, se propuso que la duración de la supervisión fuera:

- inferior o igual a 12 meses en los casos menos graves;

- comprendida entre 12 y 18 meses en los casos intermedios;

y comprendida entre 12 y 36 meses en los casos más graves.

En cuanto a las medidas aplicables en los casos menos graves, cabe mencionar que no consisten en una simple vigilancia o en un seguimiento menos intenso que el destinado a los sujetos autores de infracciones más graves. En efecto, las "líneas directrices" denominadas "nuevas penas: la ley de 2003 relativa a la justicia penal" (New sentences: Criminal Justice Act 2003), publicadas por el Consejo de líneas directrices en materia de imposición de penas (Sentencing Guidelines Council), hacen referencia a

- 40 o 80 horas de trabajo no remunerado;

- la imposición de un toque de queda;

- la prohibición durante unos meses de acudir a un determinado sitio;

la prohibición de realizar ciertas actividades; o la obligación de acudir a un determinado centro.

En caso de quebrantamiento de estas medidas, el Juez podrá:

imponer una multa de hasta 2.980 euros; modificar el contenido de la pena, imponiendo más obligaciones y prohibiciones o alargando la duración de las medidas ya fijadas.

20 Ver New sentences: Criminal JusticeAct 2003, Sentencing Guidelines Council, disponible
- revocar la sanción e imponer otra pena si la culpabilidad del autor de la infracción acaba de ser demostrada.

Si el interesado se negara a someterse a las obligaciones y prohibiciones impuestas por el Juez, este último podrá pronunciar una pena de prisión, incluso si la infracción inicial no justificaba la aplicación de una pena privativa de libertad.

\subsubsection{El Deferment of sentence}

Los artículos 1 y siguientes de la ley del año 2000 relativa a la competencia de los juzgados penales (Powers of Criminal CourtsSentencingAct 2000) prevé que el Juez podrá proceder a la suspensión de fallo de la condena durante un periodo de hasta 6 meses con el objetivo de valorar y tener en consideración la actitud del autor de la infracción una vez que haya sido declarado culpable (convicted) o los cambios que puedan intervenir en su situación personal. Este mecanismo solamente será de aplicación:

- si el delincuente da su consentimiento;

- si el delincuente se compromete a someterse a todas las obligaciones relativas a su conducta durante el periodo de suspensión (deferment) que el órgano jurisdiccional considere apropiado;

- y si el juez tiene la convicción de que el carácter de la infracción cometida, las circunstancias y la personalidad del sujeto justifican la aplicación de esta medida.

Las líneas directrices antes mencionadas indican que la medida de deferment se destina a casos pocos numerosos en los cuales "el Tribunal estaría dispuesto a imponer una pena menos severa si el interesado demostrara que está dispuesto a modificar su actitud de conformidad con las obligaciones impuestas"20.

www.sentencingcouncil.org.uk/wpcontent/uploads/web_new_sentences_guideline 1.pdf . 
En cuanto a su contenido, la orden de deferment se transmitirá a la persona designada (supervisor) para vigilar la conducta del sujeto, un agente de probation o una persona designada por el tribunal. Las líneas directrices afirman que el juez podrá imponer todas las obligaciones y prohibiciones que considera apropiadas, que sea idénticas o no a las que se aplican en caso de condena a una pena comunitaria (Community sentences).

Por fin, en caso de quebrantamiento de las medidas impuestas, el juez tendrá que volver a examinar el expediente del delincuente antes de que finalice el periodo de suspensión si este comparece ante el juzgado que solicita su comparecencia o si el juzgado tiene la certeza de que efectivamente se quebrantaron una o varias de las obligaciones impuestas.

El deferment of sentence se orienta entonces hacia el seguimiento de personas cuya situación y actitud se valora positivamente y respecto a la cuales se duda de la necesidad de aplicar medidas más estrictas y más prolongadas en el tiempo. La libertad del Juez para fijar las medidas que considera adaptadas a la situación del delincuente confiere además a este mecanismo un carácter individualizador que participa de su eficacia, al tener en cuenta la evolución de las circunstancias personales del sujeto. Cabe en consecuencia destacar la existencia en el sistema británico de dos modalidades complementarias de probatio nautónoma: el Community order, aplicable en la mayoría de los casos y que se articula alrededor de obligaciones y prohibiciones estrictamente enumeradas en la Ley pero cuya aplicación se va matizada y adaptada a cada caso gracias a "líneas directrices" y el deferment of sentence que permite alcanzar un grado más de individualización y de prevención especial al impedir la aplicación de medidas desproporcionadas.

\subsection{Suecia}

\subsubsection{Aplicación y contenido}

El capítulo 28 del Código penal sueco regula la denominada skyddstillsyn, expresión compuesta por las palabras skydd (protección, cobijo) y tillsyn (vigilancia) y que el diccionario Nordstedts traduce por "probation". Este capítulo se encuentra en la tercera parte del Código tras el capítulo 25 relativo a las multas, el 26 dedicado a la cárcel y el 27 sobre el equivalente al "sursis".

Según el art. 28.1 de este Código, el órgano jurisdiccional podrá imponer una pena de probation cuando considere que la pena de multa no constituye un castigo suficiente para el delito cometido. Su contenido podrá suponer:

la imposición de hasta 200 días-multa, se prevea o no la multa para el delito cometido; la realización de un trabajo en beneficio de la comunidad no remunerado, de un mínimo de 40 días y de un máximo de 240 horas. En este caso, el juez indicará la duración de la pena de prisión que hubiera sido impuesta si el órgano jurisdiccional hubiera elegido esta opción; la imposición de 14 días hasta 3 meses de prisión. Sin embargo, esta pena no será compatible con una multa ni con un trabajo en beneficio de la comunidad;

un programa de tratamiento denominado "contrato de cuidados" (kontraksvard) que el condenado se comprometerá a seguir. En este caso, el juez también indicará la duración de la pena de prisión que hubiera podido ser impuesta.

En la práctica, el delincuente podrá ser objeto de prescripciones particulares, impuestas con el objeto de facilitar su reinserción social y de prevenir la reincidencia. Estas obligaciones o prohibiciones guardarán relación con:

- su lugar de residencia y domicilio; 
el desempeño de un trabajo, de una actividad remunerada o de una formación;

la sumisión a un tratamiento médico.

El penado también tendrá la obligación de mantener un contacto constante con el Kriminalvarden, el servicio encargado del seguimiento de las medidas de probation.

\subsubsection{Ejecución y modificación}

La probation tendrá una duración de hasta 3 años y podrá ser acompañada por una medida de vigilancia (övervakning) a partir del día del juicio, teniendo sin embargo el juez el poder de diferir esta medida hasta la fecha en la que la sentencia se convierta en firme. Por otra parte, en caso de apelación, se podrá suspender su ejecución. Esa medida de vigilancia terminará al cabo de un año excepto en los casos en los que el condenado no respete las obligaciones que se le impusieron.

Si la probation se combina con un programa de tratamiento que el condenado se compromete a seguir, el juez podrá fijar un plazo de vigilancia específico cuya duración no podrá superar la necesaria para el desarrollo del tratamiento ni el periodo de probation de 3 años.

Cuando la probation se combina con el desempeño de un trabajo en beneficio de la comunidad, el delincuente realizará este último de acuerdo con un plan establecido por el Kriminalvarden, el servicio encargado del seguimiento de las medidas de probation. En este caso, la vigilancia se podrá prorrogar hasta la realización completa del trabajo.

Las prescripciones particulares podrán ser modificadas en función de la evolución de la situación personal del sujeto durante la ejecución de la medida. La comisión encargada del seguimiento del delincuente será la encargada de realizar estas modificaciones.

\subsubsection{Quebrantamiento}

En caso de "grave negligencia" en la ejecución del programa de tratamiento, la entidad encargada de su seguimiento tendrá la obligación de informar la Kriminalvarden, el servicio de vigilancia y el Ministerio fiscal de este quebrantamiento.

Si se vulneró una o varias de las prescripciones particulares, la comisión de vigilancia podrá avisarle del quebrantamiento de la obligación o ampliar el periodo de vigilancia hasta el final de la medida de probation, con el límite de 3 años. Si el condenado persiste en la vulneración de las obligaciones impuestas o si se supone que las nuevas medidas adoptadas quedarán sin efecto, la Kriminalvarden podrá solicitar al Ministerio fiscal la revocación de la probation. Este último también podrá actuar de oficio en los casos de grave vulneración de las medidas impuestas en el programa de tratamiento. La revocación deberá pronunciarse antes de la finalización del periodo de 3 años.

Si finalmente se revoca la probation, el juez podrá imponer otra sanción, teniendo en cuenta las medidas ya ejecutadas por el sujeto, que se trate de otra pena de probation, de una pena de prisión o de una multa. Esta nueva pena podrá consistir en una pena de prisión de duración inferior a la prevista por el Código para el delito cometido.

\section{CONCLUSIONES}

Tal y como vimos a lo largo de este trabajo, la configuración clásica de la figura de la suspensión en el ámbito europeo consiste en un modelo híbrido, que supone generalmente suspender la pena y someter al condenado a prueba. Se pretende, mediante la adopción de este modelo mixto, compensar las supuestas carencias de las figuras pioneras de la probation y del sursis.

Sin embargo, el estudio de la legislación de varios países europeos puso de manifiesto la existencia de una "pena" de probation y no de una simple 
medida alternativa a la prisión derivada de la imposición de la suspensión de la ejecución de la pena. Nos parece particularmente interesante esta figura de probation autónoma, una pena más, que no se apoya ni depende de ninguna pena de prisión y que permite afinar en mayor medida los instrumentos de individualización de los que tradicionalmente se dispone. Se trata de una figura ya implantada en varios países europeos cuya adopción deriva directamente de las circunstancias personales del sujeto, no siendo requisito imprescindible no tener antecedentes. Cabe precisar que no se trataría de eliminar o de dejar en un segundo plano la tradicional figura de la suspensión y de sustituirla por la pena de probation sino que esta última constituiría una herramienta más para imponer al delincuente la pena y medidas susceptibles de propiciar su reeducación y reinserción social.

\section{BIBLIOGRAFÍA}

Araújo, F. (2009). La suspensión como sustitutivo legal de la pena de prisión. (Tesis Doctoral) Universidad de Granada, Granada.

Barquín, J. (2007) "La suspensión condicional de la ejecución de las penas privativa de libertad desde una teoría integral del Derecho penal", material utilizado durante el Curso de Doctorado en Derecho Penal y Política Criminal de la Universidad de Granada, 19 de marzo de 2007.

Bishop, N. (2012) Le concept de "sanctions et mesures appliquées dans la communauté ", communication au colloque du 6 octobre 2012 au Sénat, sur la contrainte pénale communautaire, Arpenter le champ pénal, $\mathrm{n}^{\circ}$ 291, 15 de octubre de 2012.

Bouvier, J.C. (2013) « Une nouvelle peine au service de la probation» en AJ Pénal, marzo de 2013.
Cobo del Rosal, M.; Vives, T.(1991). Derecho penal. Parte general, $4^{\text {a }}$ Valencia, España: Tirant Lo Blanch.

Code Pénal, Dalloz, (2017).

Codice Penale, Altalex, (2017).

Dindoh, S. (2011), Sursis avec mise à l'épreuve : la peine méconnue. Une analyse des pratiques de probationen France, Direction de I' Administration pénitentiaire.

Dintilhac, J.P. (2013), «Contrainte pénale communautaire», en AJ Pénal, marzo de 2013.

Gracia, L. y Alastuey, C. (2006). Tratado de consecuencias jurídicas del delito. Valencia, España: Tirant Lo Blanch.

Herzog-Evans, M., "Récidive et surpopulation: pas de baguette magique juridique", en AJ penal, marzo de 2013.

Jescheck, H. y Weigend, T. (2003). Tratado de Derecho Penal. Parte General. Granada, España: Comares.

Judel, A. y Piñol, J.R. en Suárez-Mira (2002). Manual de Derecho penal. Parte general. Madrid, España: Civitas.

Maqueda, M.L. (1987). Algunas consideraciones sobre la teoría y la práctica de la "suspensión del fallo". III Jornadas penitenciarias andaluzas. Sevilla. España: Consejería de Gobernación.

Maqueda, M.L. (1985). Suspensión condicional de la pena y probation. Madrid, España: Centro de publicaciones del Ministerio de Justicia.

Marín de Espinosa, E. (2000). Las novedades de la suspensión de la ejecución de la pena privativa de libertad en el Código Penal de 1995. Actualidad Penal, $n^{\circ} 35$. Sin páginas 
New sentences: Criminal JusticeAct 2003, Sentencing Guidelines Council, disponible en www.sentencingcouncil.org.uk/wpcontent/uploads/webnewsentences guideline 1.pdf

Núñez, M.A. (2005). La aplicación de la probation en el derecho positivo. Suspensión del fallo y suspensión condicional de la pena. Revista de Derecho Penal y Criminología, $n^{0} 5$.

Prat, C. (2004). Alternativas a la prisión. Comentarios a las reformas introducidas por las Leyes Orgánicas 15/2003, 11/2003 y 7/2003. Madrid, España: Dykinson.
Serrano, M. (1999). Las formas sustitutivas de la prisión en el derecho penal español. Madrid, España: Trivium.

Sweden penal code, disponible en http: / /www.government. se/conte ntassets/5315d27076c942019828d6c3652 1696e/swedish-penal-code.pdf

Tournier, P., «Loi pénitentiaire, contexte et enjeux », L' Harmattan, Paris.

Tournier, P. (2013), «La contrainte pénale communautaire, créer une sanction non carcérale qui ne sera pas un sursis à l'exécution d'une sanction pénale», en Actualité juridique pénal, Dalloz. 